\title{
Portraits caricaturaux du gouvernement de Vichy sous la verve démesurée de Céline
}

Je suis descendu à Sigmaringen par patriotisme pour entendre parler le français parce que je suis un musicien. (Céline, 1980, p. 157)

Je parle de Sigmaringen, c'est un moment de l'histoire de France, (...) ça a existé et un jour on en parlera dans les écoles... (Céline, 1996, p. 937)

Accompagné de Lucette, Le Vigan et du matou Bébert, Céline rejoint Sigmaringen, transformée en ville-refuge du gouvernement de Vichy et de nombreux collaborateurs qui avaient suivi Pétain et Laval, arrivés depuis le mois d'août 1944. Céline débarque, dans ce lieu féérique et gigantesque, qui n'avait pas encore été réduit en cendres par les bombardements alliés, fin octobre 1944, après avoir traversé toute l'Allemagne du nord au sud, de la Prusse à la Bavière, puis au Bade-Wurtemberg, passant par les gares de Berlin, Leipzig, Fürth, Augsbourg, Ulm... La version officielle voulait que Céline s'y rende afin d'exercer la médecine auprès de toute la colonie française.

La description du domaine et de la ville, où « le régime vichyste achevait sa course dans la solitude glacée d'un palais délabré » (Azema \& Wieviorka, 2004, p. 314), est faite, de façon magistrale, par Jean-Paul Cointet, dans son livre, Sigmaringen, dans lequel il décrit les contours de la ville et fait un état des lieux :

Ana M. Alves - maître de conférences au Département de Langues Étrangères de l'Institut Polytechnique de Bragance - École Supérieure d’Éducation (Portugal) ; membre intégré du CLLC (Centre de Recherche en Langues, Littératures et Cultures) de l'Université de Aveiro. Adresse de correspondance : Escola Superior de Educação, Instituto Politécnico de Bragança. Quinta de Santa Apolónia. 5300-253 Bragança, Portugal ; e-mail : amalves@ipb.pt

ORCID iD : https://orcid.org/0000-0001-7762-2092 
situé en Souabe, sur le haut Danube, dans une courbe encaissée du fleuve, au cœur d'une petite ville romantique de 7000 habitants (15000 aujourd'hui), aux maisons peinturlurées de couleurs vives, aux nombreuses auberges traditionnelles, le château de Sigmaringen présente une masse imposante et inélégante, aux innombrables pièces et couloirs enchevêtrés. C'est un monstre biscornu, hérissé de tourelles, de lanternons et de pignons. (Cointet, 2003, p. 89-90)

Même si les représentations de Cointet restituent assez fidèlement cet épisode de l'histoire de France, seules la plume et la "démesure célinienne » (Combelle, 1941, cité dans Sapiro, 1999, p. 187) illustrent scrupuleusement ce scénario dans D’un château l'autre. Sous un ton de raillerie qui lui est caractéristique « au polylogue de la symphonie célinienne : musique, trame, dentelle... »(Kristeva, 1980, p. 160), Céline commence la description de Sigmaringen tout en caricaturant une population très hostile à l'égard des réfugiés français installés en ville et au château. Antinazis et solidaires des Hohenzollern, les habitants les accusaient d'être responsables de l'exil du prince et de compromettre la paix et la sécurité du bourg. En décrivant leur vie quotidienne, Céline plante le décor de sa «chronique historique » et tourne en ridicule le village de « Siegmaringen » - au lieu de « Sigmaringen » :

Peut-être pas se vanter, Siegmaringen ?... pourtant quel pittoresque séjour !... vous vous diriez en opérette... le décor parfait... vous attendez des sopranos, les ténors légers... [...] votre plateau, la scène, la ville, si jolie fignolée, rose, verte, un peu bonbon, demi-pistache, cabarets, hôtels, boutiques, biscornus pour «metteur en scène »... tout style " baroque boche " et " cheval blanc ». [...] je vous reparlerai de ce pittoresque séjour! Pas seulement ville d'eau et tourisme... formidablement historique !... Haut-lieu !... mordez Château !... stuc, bricolage [...] super-Hollywood !... toutes les époques, depuis la fonte des neiges, l'étranglement du Danube, la mort du dragon, la victoire de SaintFidelis, jusqu’à Guillaume II et Goering. (Céline, 1999, p. 156-157)

C'est bien d'une opérette qu'il s'agit, des derniers sursauts dérisoires du régime de Vichy. Céline montre qu'il n'a pas été dupe des airs d'importance que la petite colonie française a voulu se donner, en prétendant faire de Sigmaringen la capitale de la France : " alors que Pétain et Laval notamment, croient incarner la France, ils ne sont plus que des marionnettes alignées au dernier acte d'un spectacle » (Hartmann, 2006, p. 42). L'image de l'État français, esquissée par Céline, contribue « à mettre en valeur non seulement l'image d'un narrateur objectif et lucide [...] mais encore, simultanément, d'un personnage coincé par le sort, qui n'avait rien à faire là » (2006, p. 42). En effet, pour le narrateur, les jeux sont faits, le rideau tombe, la scène de l'État français est jouée :

À bien réfléchir, historique, Pétain, Debeney, étaient qui dirait, plus en scène... plus rien d'autre du tout à foutre en scène ! l'acte encore de l'« Empire Français » !... rideau ! 
aux Sénégalais ! l’acte suivant !...Pétain fini d'incarner !... la France a marre ! Qu'il rentre, qu'on le tue !... la page tourne ! (Céline, 1999, p. 193)

La représentation que fait Céline de cette ville s'apparente à une comédie, mais elle ne s'éloigne cependant pas de la chronique historique qu'il prétend faire, à partir de ses « souvenirs historiques » (Céline, 1996, p. 117) qui font de lui le « chroniqueur des Grands Guignols » (p. 732). À propos de ces circonstances historiques, Henri Godard souligne que « Céline a regagné son public en faisant un sort à une période névralgique de [l'] histoire nationale en mettant en avant un rôle modeste de chroniqueur » (Godard, 1985, p. 379). À cette affirmation, il ajoute qu' " en réalité, ces évènements si haut proclamés "historiques", il les traite sans plus de souci d'exactitude que le reste de son expérience " (p. 379). Godard achève cette réflexion en assurant que « du chroniqueur, Céline n'a ni le ferme propos de fidélité aux faits, ni le respect de l'ordre chronologique, ni l'effacement de sa personne devant la grandeur des événements auxquels il assiste» (p. 379).

Au sujet de cette reconstitution de l'histoire, Hannah Arendt est d'avis que

le but de ces reconstructions variées et d'ailleurs variables était toujours de dénoncer l'histoire officielle comme une farce, de mettre au jour une sphère d'influences secrètes dont la réalité historique visible, vérifiable et connue n’était qu’une façade explicitement plantée pour tromper le peuple. (Arendt, 1973, p. 81)

Ainsi, entre l'humour, «le dégoût et le rire, l'apocalypse et le carnaval » (Kristeva, 1980, p. 161), le narrateur renforce le statut que les allemands avaient attribué à cette colonie française :

la Chancellerie du Grand Reich avait trouvé pour les Français de Sigmaringen une certaine façon d'exister, ni absolument fictive, ni absolument réelle, qui sans engager l'avenir, tenait tout de même compte du passé... statut fictif, « mi-quarantaine mi-opérette » [...] finalement nous étions reconnus à titre précaire-exceptionnel « réfugiés en enclave française » à condition de...de...tout de même en enclave française! (Céline, 1999, p. 333-334)

Sous un ton de moquerie, de détails " comiques, risibles ou grotesques " (Mikoff, 1999, p. 209), auxquels il nous a habitué, Céline nous présente certains portraits caricaturaux des habitants de cette enclave. Les réfugiés miliciens, collaborateurs et leurs familles sont installés dans différents endroits de la ville, non loin du château, qu'ils nomment "l'Olympe ». Dans cette demeure, au septième étage, dans les appartements du prince on retrouve le maréchal, sa femme et le docteur Ménétrel, médecin et conseiller du maréchal qui sera, très tôt, écarté de Sigmaringen par les Allemands, jugeant qu'il dissuadait Pétain de la collaboration souhaitée comme y fait référence Henri Godard dans la notice D'un château l'autre (Céline, 1996, p. 982). 
Le Maréchal Pétain, ou bien l'« Incarneur total (Céline, 1999, p. 192), comme le baptise Céline, est d’emblée condamné au début du récit : « Merde !... ils ont bien fait de le buter !... Verdun, patati et patata !... je l'ai connu [...] à Siegmaringen, je sais ce que je cause » (p. 28).

Céline met également en scène dans D'un château l'autre Pierre Laval, ancien Président du Conseil vichyste, hébergé au sixième étage, avec son épouse. Par le biais du mode satirique, il apparaît à deux reprises dans la gare de Sigmaringen et dans l'appartement qui lui a été attribué au château. Céline commence par faire un tableau flatteur de son attitude, lorsqu'il se retrouve mêlé à une scène de cohue à la gare : « il était brave... il haïssait les violences... Laval était le conciliant né... le Conciliateur !... et patriote !... et pacifiste !... moi qui voit que des bouchers partout !... lui pas ! pas !... pas !» (Céline, 1999, p. 254). Cet enthousiaste portrait est rapidement privé de son ardeur et de sa grâce lorsque dans un entretien avec l'ancien Président du Conseil, auquel se joindra l'ex-ministre Jean Bichelonne, «l'un des rares avec qui Céline sympathisera et qui avait été chargé à Vichy du secrétariat d'État à la Propagande » (Vitoux, 1988, p. 420), Laval commence une longue plaidoirie qui ne permet aucune intervention au narrateur de D'un château l'autre. N'acceptant pas son rôle de simple auditeur, ce dernier se sent tenté d'ébaucher quelques réflexions railleuses à son sujet, accentuant l'arrogance du personnage et faisant des commentaires racistes à son égard :

il en savait bien plus que moi !... bien sûr !... il en savait bien plus que tout le monde... en tout !... et sur tout! Bicot, avec sa mèche débène, il lui manquait que le fez crasseux... il était le vrai bicot de III qui parle à tous les voyageurs, qui sait mieux que tous ceux qui sont là ce qu'ils devraient faire. (Céline, 1999, p. 352)

Il me tolérait comme auditeur ! pas commentateur !... Je rengaine donc mes compliments...[...] je connaissais sa plaidoirie... dix... vingt fois il me l'avait servie !... [...] je connaissais toutes les variantes, feintes, objections, appels pathétiques... [...] que lui Laval, pas à confondre ! que lui, avait la France dans le sang !... qu'il faudrait bien qu'ils l'avouent, gnomes imbéciles. (p. 351)

Soulé par les paroles d'un si long discours, le narrateur parvient à engager le dialogue. Cependant, le ton, déjà en phase de transformation, passe au stade des injures. Dans cette scène, le narrateur met en évidence le physique de gitan de Laval. De son côté, l'ancien Président lui rappelle les propos racistes qu'il a tenus à son égard :

Il m'attendait au détour... il m'envoie sa botte ! « Vous êtes d'accord avec un juif ? » Ça y est !... le mot! Le mot juif !... c'était fatal qu'il m'en parle ! la vache il attendait le monde ! Il prend l'offensive... « Vous m'avez bien traité de juif, n'est-ce pas Docteur ? » Oui, je le sais !... pas que vous! Je suis partout aussi ! - Eux, pas tout à fait, Monsieur le Président! (p. 354) 
À propos de cet extrait, Christine Sautermeister affirme qu'il " apparaît comme un règlement de comptes de Laval, le narrateur maintient ses dires tout en se distanciant du journal vichyssois Je suis partout, accusé de complaisance vis à vis de Laval » (Sautermeister, 2002, p. 307). Sautermeister ajoute, à ce sujet, que nous sommes en présence d'un "Céline critique vis à vis de Vichy mais qui affiche son propre racisme. L'écrivain contre-attaque, se plaignant dềtre à Sigmaringen par sa faute. Il accuse Laval de ne pas avoir tenté, comme il le fit pour "la bande", de le "caser ailleurs" " (Céline, 1999, p. 355). Il en profite pour lui faire sentir son mécontentement :

Je prends la moutarde! merde! ces airs « de ne pas savoir »! Je sais ce que je dis !... il serait bien content, bicot torve, que je paye pour la bande ! Que j'écope pour la compagnie ! Fripouilles, connivents, triple-jeux ! L'addition pour ma cerise! (p. 355)

Après la présentation caricaturale de Laval, un autre personnage entre en scène ; il s'agit de Jean Bichelonne, ancien ministre de l'industrie qui n’échappe pas, lui non plus, à l'œil vif du narrateur, qui le présente comme celui qui avait « la plus grosse tête, pas seulement qu'il était champion de Polytechnique et des Mines... Histoire ! Géotechnie !... pardon !... un vrai cybernétique tout seul !» (Céline, 1999, p. 157). Il s'agit, d'après le narrateur, d'un "spermatozoïde monstre » (p. 357) au vu de son « énorme tronche» (p. 357), de sa «formidable tronche» (p. 159). Comme le souligne Anne Henry, la description de ce "polytechnicien surdoué dépassé par l'évènement " (1994, p. 234) est, bien évidemment, marquée par une exagération bien célinienne.

Au-delà de ces «privilégiés » de la Collaboration, aperçus par Céline à Sigmaringen, l'auteur expose les conditions de vie des simples réfugiés, des "vilains » dépossédés de leurs biens, de leurs illusions. Céline introduit, à nouveau, dans le récit qu'il fait de son expérience à l'intérieur de ce village bavarois, un thème qui lui est cher et qui consiste, selon ce que nous décrit Henri Godard dans la notice de D'un château l'autre, dans

la division de l'humanité [...] entre riches et pauvres ; les premiers mangent, boivent et jouissent, et prêchent aux seconds, qui travaillent et meurent de faim, toutes les vertus d'abstinence et de résignation. Sigmaringen semble d'abord évoqué pour illustrer cette division : d'un côté les "gâtés du château ", nourris, lavés et chauffés, de même que les curés du Fidelis; de l'autre « les ports galeux ». (Céline, 1996, p. 968)

Ces derniers sont entassés dans la petite ville, que ce soit dans la gare, où ils attendent un hypothétique train, soit dans un logement, dans l'une des auberges bondées comme le Bären ou le Löwen où vivent Céline, Lucette et Bébert au premier étage, chambre 11. Les femmes et les enfants des miliciens sont internés dans le camp que Céline désigne comme Cissen, mais qui est, en réalité, le camp de Biessen, qui ressemble à un camp de concentration. Ils y survivent dans des conditions déplorables, 
au milieu d'une grande insalubrité où ils ont « vraiment très crevé de faim » (Céline, 1996, p. 160).

Alors que le rationnement est très strict pour les réfugiés qui sont parqués hors du château, à l'intérieur de l'immense bâtisse, au contraire, la nourriture est abondante. Pétain, par exemple, dispose de « seize cartes » d'alimentation qu'il garde de façon égoïste. Céline démystifie cruellement son prestige et marque l'inégalité redoublée face à ceux qui doivent presque quémander un peu de pain. Il profite également de cette inégalité pour souligner non seulement son inimitié envers Pétain, mais surtout pour ridiculiser les thèmes de la Révolution Nationale : "Famille, Travail, Patrie ? Merde !... [...] je l'ai connu avec ses seize cartes à Siegmaringen, ... » (p. 28).

Tourmenté par les problèmes de ravitaillement, Céline achetait au marché noir. Outre cette obsession pour les provisions, il souffrait d'un délire de persécution se trouvant partout une foule d'ennemis imaginaires, constamment à l'affût des moindres indices de machinations dressées contre lui. Lucien Rebatet, qui était également réfugié à Sigmaringen et qui a fréquenté Céline au quotidien jusqu'au départ de celui-ci en mars 1945, affirme que ces traits d'obsession et de persécution auraient pu devenir intolérables. Toutefois, lorsqu'il était en milieu français, écarté de ces problèmes, il retrouvait sa fougue et sa gaieté, il amusait son auditoire. Dans D'un Céline l'autre, Lucien Rebatet, qui faisait partie de son petit cercle, raconte quelques moments de détente :

satisfait de sa manœuvre, de nos rires, il [Céline] s'engageait dans un monologue inouï, la mort, la guerre, les armes, les peuples, les continents, les tyrans, les nègres, les jaunes, les intestins, le vagin, la cervelle, les Cathares, Pline l’ancien, Jésus-Christ. La tragédie ambiante pressait son génie comme une vendange. Le cru célinien jaillissait de tous côtés. Nous étions à la source de son art. (Rebatet, 1963, p. 52)

Céline n'épargnait pas ses remarques sarcastiques aux Allemands : il leur en voulait d'avoir perdu la guerre. Cependant, les hôtes allemands se montraient très tolérants à son égard, car ils admiraient le grand écrivain. Lucien Rebatet raconte un dîner auquel assistaient de hautes personnalités :

De nombreuses autorités militaires et administratives du « Gau » sétaient fait inviter, friandes d'un régal d'esprit parisien. Il y avait même un général, la « Ritterkreuz au coup ». Céline, qui ne buvait pas une goutte de vin, entama un parallèle opiniâtre entre le sort des « Friquets », qui avaient trouvé le moyen de se faire battre, mais pour rentrer bientôt chez eux, bons citoyens et bons soldats, consciences nettes, ne devant des comptes à personne, ayant accompli leur devoir patriotique, et celui des " collabos " français qui perdaient tout dans ce tour de cons, biens, honneur et vie. Alors lui, Céline, ne voyait plus ce qui pourrait l'empêcher de proclamer que l'uniforme allemand, il l'avait toujours eu à la caille, et qu'il n'avait tout de même jamais été assez lourd pour se figurer que sous 
un pareil signe de collaboration ne serait pas un maléfice atroce. Mais les hauts militaires avaient décidé de trouver la plaisanterie excellente... (p. 53)

Le peuple allemand est, aux yeux de Céline, à l'image de ses lieux de vie : lourd, triste et ridicule. En outre, les Allemands se divisent globalement en deux groupes : les nazis, froids, efficaces et courtois, mais animés d'un délire sanguinaire, et les antinazis, souvent des nobles prussiens, parfois amicaux. Quelques-uns sont hostiles à Céline, telle la doctoresse de Sigmaringen qui refuse de l'aider à soigner un malade français. À son tour, Céline, ne leur fait pas confiance : « les fritz sont sournois perfides !... vous pouvez vous attendre à tout ! regardez d'abord les music-halls, tous les prestidigitateurs sont boches ! [...] ils sont à se méfier terrible !...» (Céline, 1999, p. 185).

Après les remarques esquissées sur les allemands, le narrateur D'un château l'autre décrit des évènements, qui sont purement imaginaires, comme la promenade de Pétain au bord du Danube, les émeutes devant le château et dans la gare, ou encore le dîner chez Abetz, ambassadeur d'Allemagne à Paris sous l'Occupation, qui prendra, dans le récit de Céline, le nom de « limogé Abetz [celui qui] donnait encore et malgré tout, tantôt ici tantôt là, des sortes de "surprise-partys" " (Céline, 1999, p. 333). Chargé à Sigmaringen de veiller à l'installation des réfugiés, Abetz a reçu " la mission de persuader Pétain et Laval de renoncer à leur passivité. Trois mois lui avaient été accordés par Ribbentrop pour atteindre son but. Ayant échoué, il fut révoqué [...] et remplacé par un nouvel ambassadeur, von Reinebeck» (p. 982). Pris de délire nazi, Otto Abetz est décrit comme un illuminé dont le plus grand "projet auquel il tient » (p. 339) est la célébration de « sa grande œuvre, [...] la plus colossale statue, Charlemagne en bronze, en haut de l'avenue de la Défense !» (p. 339), « la très grande symbolisation que toute l'Europe attendait! » (p. 340). C'est alors que Céline met en scène une dispute entre Abetz et l'écrivain français, Alphonse de Châteaubriant, sur le choix d' " une Ode » (p. 340) qui devrait être jouée lors de l'inauguration de cette "formidable statue, dix fois plus grosse, large, haute, que la "Liberté" de New York! » (p. 343). Déconcerté par les propositions de Abetz, qu'il traite de " peigne-cul » (p. 344), Châteaubriant explose " regarde la table... attrape une soucoupe... et vlang ! Y envoie !... et encore une autre !... et une assiette !...et un plat !... c'est la fête foraine ! [...] partout ! Et encore ! C'est du jeu de massacre! » (p. 344).

Au sujet de ce portrait caricatural de Abetz, nous aimerions mettre en avant l'analyse proposée par Marie Hartmann qui défend que Céline « raille l'élaboration d'un passé mythique servant de prétexte aux destructions présentes » (Hartmann, 2006, p. 121). Encore une fois, pour justifier son dessein, Céline utilise l'injure, l'irréel. Comme le précise Karl Epting, cette rencontre «n’a [...] jamais pu avoir lieu, ne serait-ce que parce que Abetz n'a presque jamais franchi le seuil du château " (Epting, 1963, p. 57). Epting ajoute même que Abetz lui a confié, peu avant sa mort, « combien certaines réalités intérieures de cette époque étaient devenues transparentes 
dans cette scène, par le grotesque même des situations et des personnages, $y$ inclus sa propre personne» (p. 57).

Au terme de cette analyse, et comme le défend Eric Mazet, « tout en n'ayant cessé d'arracher les masques de ses contemporains, de mettre à nu sa chair et ses nerfs, Céline aura été un fantastique comédien de sa propre existence et le formidable créateur de personnages de comédie " (Mazet, 1993, p. 211). De la sorte, nous pouvons affirmer qu’afin de donner plus de force littéraire à son récit,

Céline soumet chaque événement à la technique de la transposition, du « rendu émotif ». Il déforme les évènements au même degré qu'il déforme la langue, et dans le même souci "d'impressionnisme ». C'est pourquoi on le trouve menteur, hypocrite, bas, etc. En faisant déjà dans les écrits intimes ce qu'il fera dans ses romans, il ne met pas de frontières entre son existence et son écriture. (Céline, 1980, p. 190-191)

Comme le souligne si bien François Gibault, Céline « reste fidèle à sa technique de transposition qui consistait à ne jamais se contenter du mal et à rechercher toujours le pire» (1981, p. 289). Il nous semble que sur l'univers célinien pèse « le glaive invisible d'un jugement " (Kristeva, 1980, p. 162). Glaive, qui «n'est peut-être même pas une instance mais une distance : [...] un détachement qui f[ait] exister l'horreur et en même temps nous en écart[e]» (p. 162). Glaive qui "nous saisi[t] d'effroi, et de cette frayeur même font du langage une plume, fuyante, perçante, une dentelle, voltige, éclat de rire et note de mort» (p. 162).

"Il faut être plus qu'un petit peu mort », comme l'affirme d'ailleurs Céline dans les Entretiens au professeur $Y$, " pour être vraiment rigolo ! Voilà ! Il faut qu'on vous ait détaché » (Céline, 1993, p. 519). Idée qu'il développe dans une lettre à Milton Hindus, datée du 31 mars 1948 : "Je suis "détaché", sérieux, classique dans mon délire - constructif - par-là peut-être je me rapproche des grands - c'est tout... » (Hindus, 1969, p. 186). Ce délire célinien avait déjà été caractérisé par Combelle lorsqu'il affirmait en 1941: "la démesure célinienne veut que le lecteur ait bon estomac et bon esprit. Son génie touche esprit et corps. C'est pourquoi il est sain en dépit de sa boue » (Combelle, 1941, cité dans Sapiro, 1999, p. 187). Dans Féerie pour une autre fois $I$, Céline renforcera cette posture délirante qui définit son "identité littéraire " (Roussin, 2005, p. 24) et que l'on retrouvera dans toute son œuvre : « J'ai des visions? ... j'ai l'ouie fantasque ? [...] c'est manière de rire ! pas plus ! [...] j'ai le rire naturel... de l'embellie dans la vacherie... c'est pas tout le monde...! [...] le texte vous vexe ? ça vous regarde !» (Céline, 1993, p. 27). 


\section{RÉFÉRENCES}

Arendt, H. (1973). Les Origines du totalitarisme, Sur l'antisémitisme. Paris : Calmann-Lévy. Azema, J. P. et Wieviorka, O. (2004). Vichy 1940-1944. Paris : Perrin.

Céline, L. F. (1980). Lettres à Albert Paraz, 1947-1957. J.-P. Louis (éd.). Dans Cahiers Céline 6. Paris : Gallimard.

Céline, L. F. (1993). Féerie pour une autre fois I et II, Entretiens avec le professeur Y, Romans t. IV. H. Godard (éd.). Paris : Gallimard.

Céline, L. F. (1996). D’un château l'autre, Nord, Rigodon. Romans, t. II. H. Godard (éd.). Paris : Gallimard.

Céline, L. F. (1999). D’un château l'autre. Paris : Gallimard.

Cointet, J.-P. (2003). Sigmaringen, une France en Allemagne (septembre 1944-avril 1945). Paris : Perrin.

Epting, K. (1963). Céline ne nous aimait pas. Dans L. F. Céline, Cahiers de L'Herne, no 3 (p. 5659). Paris : L'Herne.

Gibault, F. (1981). Céline III, Cavalier de l’Apocalypse, 1944-1961. Paris : Mercure de France.

Godard, H. (1985). Poétique de Céline. Paris : Gallimard.

Hartmann, M. (2006). L'envers de l'histoire contemporaine. Étude de la "trilogie allemande" de Louis-Ferdinand Céline. Paris : Société des études céliniennes et Marie Hartmann.

Henry, A. (1994). Céline écrivain. Paris : L'Harmattan.

Hindus, M. (1969). L. F. Céline tel que je l'ai vu. Paris: L’Herne.

Kristeva, J. (1980). Pouvoirs de l'horreur. Paris : Seuil.

Mazet, E. (1993). D’un masque l’autre. L’Année Céline 1993, 211-222.

Mikoff, I. (1999). L'illusion comique célinienne. Dans Classicisme de Céline. Actes du douzième Colloque international Louis-Ferdinand Céline, Abbaye d’Ardenne, July 3-5, 1998 (p. 209-226). Paris : Société d'études céliniennes.

Rebatet, L. (1963). D’un Céline l'autre. Dans L. F. Céline, Cahiers de L’Herne, no 3 (p. 42-55). Paris : L'Herne.

Roussin, P. (2005). Misère de la littérature, terreur de l'histoire. Céline et la littérature contemporaine. Paris : Gallimard.

Rousso, H. (1984). Pétain et la fin de la collaboration, Sigmaringen 1944-1945. Bruxelles : Complexe.

Sapiro, G. (1999). La guerre des écrivains (1940-1953). Paris : Fayard.

Sautermeister, C. (2002). Céline Vociférant ou l'art de l'injure. Paris : Société des études céliniennes et Christine Sautermeister.

Vitoux, F. (1988). La vie de Céline. Paris : Bernard Grasset.

RÉSUMÉ : Dans ses trois romans, communément désignés comme trilogie allemande, Céline évoque le récit de son périlleux exode en Allemagne, de 1944 à 1945, puis au Danemark de 1945 à 1951. Notre propos n'est pas de reprendre tout ce périple, mais de nous attarder sur son séjour à Sigmaringen où presque toutes les figures marquantes de la Collaboration sont dépeintes et caricaturées par Céline sur un ton de mépris qui lui est exclusif et marque sa singularité exceptionnelle. Nous en retiendrons 
quelques exemples pour montrer comment l'écrivain amplifie les traits de ses personnages, pour donner plus de vigueur à ses descriptions. Céline brosse leurs portraits, en accentuant leurs caractères ou bien en leur prêtant, parfois, des attitudes ridicules. Par des détails hilariants, burlesques, comiques, Céline parvient à caricaturer certaines personnalités du gouvernement de Vichy dans une atmosphère où se mêlent absurdité, chaos, décadence.

Mots-clés : Céline, Sigmaringen, caricature, comique, grotesque, démesure

\section{Caricatural portraits of the Vichy government under Céline's inordinate verve} ABSTRACT: In his three novels, commonly referred to as the German trilogy, Céline evokes the story of his dangerous exodus to Germany, from 1944 to 1945, then to Denmark from 1945 to 1951 . Our purpose is not to take the whole journey back, but to dwell on his stay in Sigmaringen where almost all the outstanding figures of the Collaboration are depicted and caricatured by Céline in a tone of contempt particular of him and of his exceptional singularity. We will use a few examples to show how the writer amplifies his characters' features, to strengthen his descriptions. Céline paints their portraits, exaggerating their characteristics or, sometimes, endowing them with ridiculous attitudes. By hilarious, burlesque, comical details, Céline manages to caricature certain figures of the Vichy government in an atmosphere where absurdity, chaos and decadence mingle.

Keywords: Céline, Sigmaringen, caricature, comic, grotesque, excess 